\title{
RESEARCH
}

Open Access

\section{Adult and iPS-derived non-parenchymal cells regulate liver organoid development through differential modulation of Wnt and TGF- $\beta$}

Ernesto Goulart ${ }^{1}$, Luiz Carlos de Caires-Junior ${ }^{1}$, Kayque Alves Telles-Silva', Bruno Henrique Silva Araujo², Gerson S. Kobayashi', Camila Manso Musso', Amanda Faria Assoni' ${ }^{1}$, Danyllo Oliveira', Elia Caldini Jonathan A. Gerstenhaber ${ }^{4}$, Silvano Raia ${ }^{5}$, Peter I. Lelkes ${ }^{4}$ and Mayana Zatz ${ }^{1 *}$ (D)

\begin{abstract}
Background: Liver organoid technology holds great promises to be used in large-scale population-based drug screening and in future regenerative medicine strategies. Recently, some studies reported robust protocols for generating isogenic liver organoids using liver parenchymal and non-parenchymal cells derived from induced pluripotent stem cells (iPS) or using isogenic adult primary non-parenchymal cells. However, the use of whole iPSderived cells could represent great challenges for a translational perspective.

Methods: Here, we evaluated the influence of isogenic versus heterogenic non-parenchymal cells, using iPSderived or adult primary cell lines, in the liver organoid development. We tested four groups comprised of all different combinations of non-parenchymal cells for the liver functionality in vitro. Gene expression and protein secretion of important hepatic function markers were evaluated. Additionally, liver development-associated signaling pathways were tested. Finally, organoid label-free proteomic analysis and non-parenchymal cell secretome were performed in all groups at day 12 .

Results: We show that liver organoids generated using primary mesenchymal stromal cells and iPS-derived endothelial cells expressed and produced significantly more albumin and showed increased expression of CYP1A1, CYP1A2, and TDO2 while presented reduced TGF- $\beta$ and Wnt signaling activity. Proteomics analysis revealed that major shifts in protein expression induced by this specific combination of non-parenchymal cells are related to integrin profile and TGF- $\beta /$ Wnt signaling activity.
\end{abstract}

Conclusion: Aiming the translation of this technology bench-to-bedside, this work highlights the role of important developmental pathways that are modulated by non-parenchymal cells enhancing the liver organoid maturation.

Keywords: Organoid, Liver, iPS, Hepatocyte, 3D culture

\footnotetext{
* Correspondence: mayazatz@usp.br

${ }^{1}$ Human Genome and Stem-Cell Research Center (HUG-CEL), Department of Genetics and Evolutionary Biology, Institute of Biosciences, University of São Paulo, São Paulo, SP, Brazil

Full list of author information is available at the end of the article
}

(c) The Author(s). 2019 Open Access This article is distributed under the terms of the Creative Commons Attribution 4.0 International License (http://creativecommons.org/licenses/by/4.0/), which permits unrestricted use, distribution, and reproduction in any medium, provided you give appropriate credit to the original author(s) and the source, provide a link to the Creative Commons license, and indicate if changes were made. The Creative Commons Public Domain Dedication waiver (http://creativecommons.org/publicdomain/zero/1.0/) applies to the data made available in this article, unless otherwise stated. 


\section{Background}

Liver organogenesis can be in part recapitulated in part by using organoid technology [1]. The combination of defined ratio of parenchymal progenitor cells (i.e., hepatoblast) and non-parenchymal cells (NPC) (i.e., endothelial cells and mesenchymal cells) recreates a cellular microenvironment akin to the early stages of liver bud development and allows for spontaneous tissue formation [2]. The first attempts to bioengineer complex liver organoids (LOs) used hepatoblasts derived from human pluripotent stem cells (iPS) in conjunction with primary human NPC, such as human umbilical cord-derived endothelial cells (HUVEC) and adipose tissue-derived mesenchymal stem cells (MSCs), all derived from different donors [1].

NPC contribute to liver development and homeostasis by secreting growth factors (e.g., TNF- $\alpha$, IL-6, HGF, TGF- $\beta$, and BMP2, 4 and 6) that regulate hepatocyte proliferation, DNA synthesis, and hepatic cord formation [3-5]. Asai and collaborators [6] showed the distinct contributions of primary lineages of endothelial cells (ECs) and MSC secretome in LO development in vitro. More recently, some other groups reported a series of combined protocols for generating isogenic LOs obtained from whole iPS-derived cells, obtained from the same donor, or by using primary NPCs from the same donor [7-9]. Takebe and collaborators [7] successfully generated LOs from human donors that could potentially be applied for high-throughput personalized screening of liver toxicity.

However, large-scale differentiation of iPS into multiple cell lineages is challenging in terms of cost and efficiency as opposed to primary cell lineages. As a caveat, the use of standard commercial non-parenchymal cell lines will yield human LOs that are chimeric in nature. Here, we propose to evaluate the effects of applying liver NPCs derived from iPS-derived fetal-like cells versus adult primary NPC cell lines to LO development and functionality.

\section{Methods}

iPS generation and culture and primary adult cell culture Induced pluripotent stem cells (iPSs) were generated from three healthy human donors (F9048=male, 26; F8799 = female, 28; F7405 = male, 23), as previously described [10]. The reprogramming and cell culture protocol were described in Additional file 1: methods. Differentiation protocols and human primary adult cell culture methods were described in Additional file 1: methods.

\section{Liver organoid}

Prior to cell seeding, Matrigel was diluted 1:1 on ice with cold EGM-2 and dispensed at $380 \mu \mathrm{L} /$ well in a 24-well plate. Gelling was achieved by incubation in $37^{\circ} \mathrm{C}$ for at least $30 \mathrm{~min}$. A mixture of iPS-derived cells $\left(1 \times 10^{6}\right.$ hepatoblast, $8 \times 10^{5} \mathrm{ECs}$, and $2 \times 10^{5} \mathrm{MSCs}$, as per Takebe et al. [1]) was centrifuged for $5 \mathrm{~min}$ at $300 \times g$ and resuspended in $2 \mathrm{~mL}$ of LO culture media (composed of 1:1 EGM-2/hepatocyte differentiation media, see Additional file 1: methods). The cell mixture was seeded on top of the Matrigel bed. Media was changed every other day. In order to assess the rate of mesenchymal condensation, pictures of the wells were taken every $12 \mathrm{~h}$. The confluent cell layer and the total covered area progressive condensation over time was evaluated using ImageJ software.

\section{Proteomics}

Proteomic sample processing and analysis followed a previously published protocol [11]. For detailed information, see Additional file 1: methods section. Pathway annotation of protein IDs was performed using the comprehensive EnrichR gene set enrichment analysis web server [12, 13], applying Reactome [14] and Panther [15] categorization with the significance threshold set at $p<0.05$. Interactome analysis was performed using String [16] with k-means clustering in three groups.

\section{Statistical analysis}

Statistical analyses to assess LO functional analysis and development quality (Figs. 2 and 4) were performed using one-way ANOVA with Tukey's post-test. For all other statistical analyses, Student's two-tailed $t$ tests were used for pairwise comparisons. Data are presented as means \pm SEM, or mean of at least three independent experiments, with at least two technical replicates. For the proteomics analyses, statistical tests were performed using Students $t$ test, using Perseus software, and pathway enrichment analysis using EnrichR. Values of $p<$ 0.05 were considered significant. GraphPad Prism software was used to perform all other statistical analyses.

\section{Results}

\section{iPS cell differentiation}

Aiming a broad applicability of our studies and reproducibility of the results, we carried out the experiments with three independent iPS cell lines. All data shown in Fig. 1 are related to cell line F9048, and similar results were observed with the other cell lines (Additional file 1: Figure S1C). Figure 1a summarizes the groups tested in this study, using different combinations of NPCs with iPS-derived hepatocytes. Here, we have used "I" to indicate cells derived from iPS and "P" to indicate primary cell lines. Isogenic LO (i.e., containing all three cell lines derived from the same iPSs) is referred to as III. When using NPC derived from primary human cell lines, the group is referred to as IPP. When using human aortic 


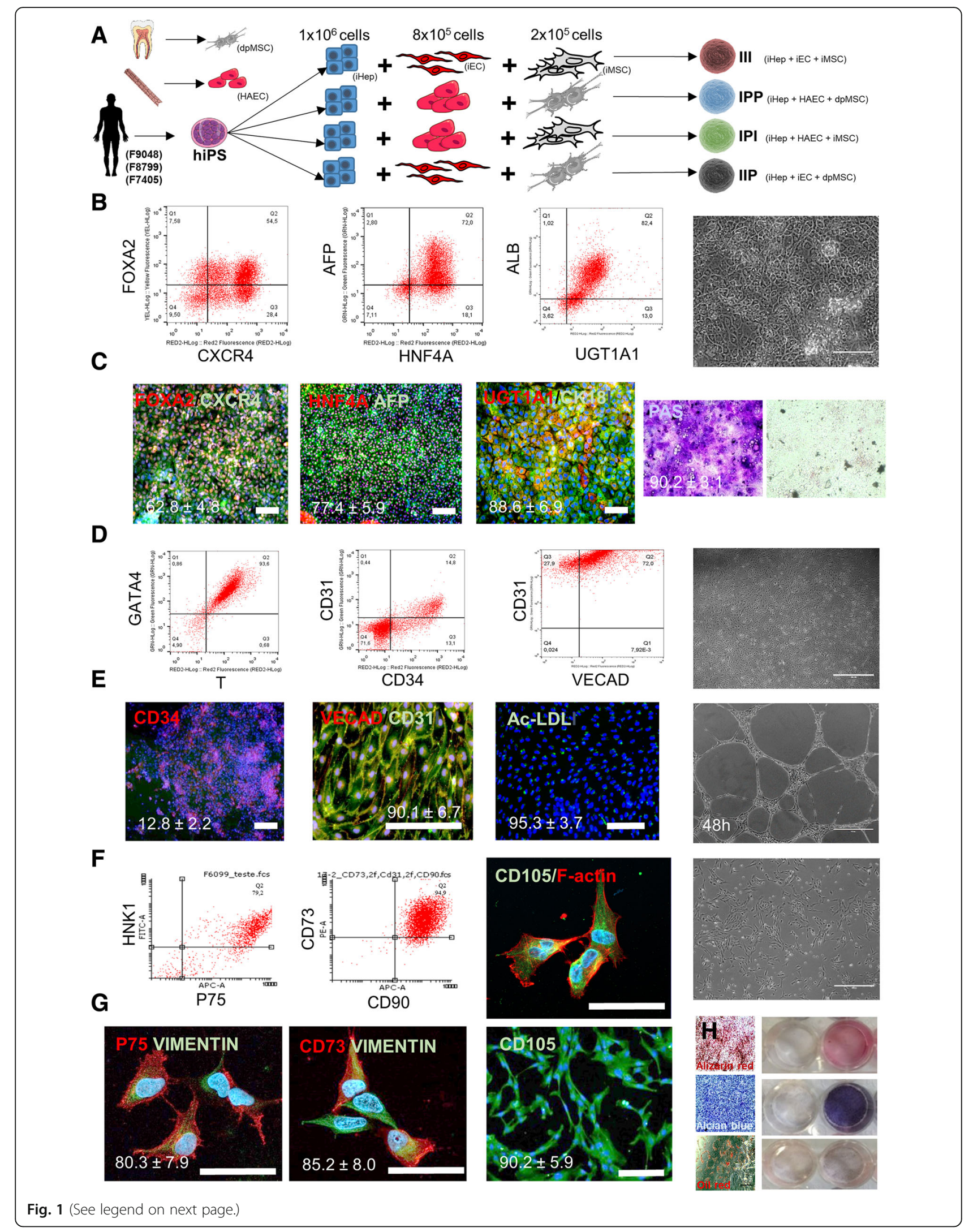


(See figure on previous page.)

Fig. 1 iPS cell differentiation. a Graphical summary of experimental groups and design. b Step-wise flow cytometry characterization of hepatocyte differentiation in vitro. Representative image of gated analyses for FOXA2 $2^{+} / \mathrm{CXCR} 4^{+}$at day $3, \mathrm{AFP}^{+} / \mathrm{HNF}_{\mathrm{AN}}{ }^{+}$at day $9, \mathrm{UGT1A1}^{+} / \mathrm{ALB}^{+}$at day 18, and phase-contrast image of cellular morphology at day 18. c IF staining for FOXA2/CXCR4 at day 3, AFP/HNF4A at day 9, and UGT1A1/ ALB and PAS staining of hepatocytes at day 18 and confluent fibroblast culture PAS staining negative control $(n=3$, biological replicates; data displayed as mean \pm SEM, bar $=50 \mu \mathrm{m}$ ). $\mathbf{d}$ Step-wise flow cytometry characterization of endothelial differentiation in vitro. Representative images of gated analyses for BRACHYURY ${ }^{+} / \mathrm{GATA}^{+}$at day $2, \mathrm{CD} 4^{+} / \mathrm{CD} 31^{+}$at day $6, \mathrm{VECAD}{ }^{+} / \mathrm{CD} 31^{+}$at day 10 , and phase-contrast image of cellular morphology at day 10. e IF staining for CD34 at day 2, VECAD/CD31 and AC-LDL uptake at day 10, and angiogenesis assay at day $12(n=3$, biological replicates; data displayed as mean \pm SEM, bar $=50 \mu \mathrm{m})$. $\mathbf{f}$ Step-wise flow cytometry characterization of iNCC-MSC differentiation in vitro. Representative image of gated analyses for P75+/HNK1+ at day 18 and CD73+/CD90+ and IF staining for CD105/F-Actin and phase-contrast image at day 28. $\mathbf{g}$ IF staining for P75NIMENTIN at day 18, CD73/NIMENTIN and CD105 at day 28, and representative images for MSC differentiation assay after 28 days of induction and stained for Alizarin Red, Alcian Blue, and Oil red ( $n=3$, biological replicates; data displayed as mean \pm SEM, bar $=50 \mu \mathrm{m}$ )

endothelial cells (HAECs) and iPS-derived MSC, the group is referred as IPI. Finally, when using iPS-derived EC and dental pulp-derived MSC (dpMSC), the group is referred as IIP.

The hepatic differentiation potential was evaluated and characterized in vitro. Figure $1 \mathrm{~b}$ shows representative images of flow cytometric analyses. After 3 days of differentiation, $58.5 \pm 4.7 \%(n=3)$ of cells were $\mathrm{CXCR}^{+} /$ FOXA2 ${ }^{+}$(definitive endoderm). Despite starting out with a heterogeneous population, at day 9 of differentiation, a majority of cells $(78 \pm 5.8 \%, n=3)$ expressed hepatic progenitor markers, such as HNF4A and AFP. After terminal hepatocyte differentiation, $74.3 \pm 7.1 \%(n=3)$ of the cells expressed the hepatic markers ALB and UGT1A1 (Fig. 1b). Phase-contrast image showed homogenous hepatocyte morphology in a monolayer culture. Representative images of immunofluorescence (IF) staining for each step of hepatic differentiation are shown in Fig. 1c. After 3 days, $62.8 \pm 4.8 \%(n=3)$ of cells were double positive for FOXA2 and CXCR4. After 9 days, $77.4 \pm 5.9 \%(n=3)$ were positive for HNF4A and AFP, and at day $18,88.6 \pm 6.9 \%(n=3)$ of cell population were positive for ALB and UGT1A1. Also, at day 18, $90.2 \pm 3.1 \%(n=3)$ cells stained positive for Periodic acid-Schiff (PAS) (Fig. 1c).

For assessing endothelial differentiation, the iPS-derived cells were evaluated stepwise throughout the differentiation protocol. Figure 1d shows representative images of the flow cytometric analyses. The first step of the differentiation protocol (day 2) resulted in homogenous mesodermal differentiation, as inferred from the nearly ubiquitous co-expression of Brachyury $\mathrm{T}$ and GATA4 $(92.4 \pm 3.7, n=3)$. However, at the end of endothelial differentiation (day 7), only $13.1 \pm 2.7 \%$ of the cell population was double positive for endothelial markers CD34 and CD31. After magnetic sorting of the CD31+ cells and seeding a $60 \%$ confluent cell culture, the great majority of cells were positive for CD31 and a varying percentage of them were also positive for VECAD $(48.6 \pm 22.8 \%, n=3)$. At day 7 of endothelial differentiation, $12.8 \pm 2.2 \%$ of cells were positive for CD34.
After cell sorting, $90.1 \% \pm 6.7 \%$ of cells were double positive for CD31 and VECAD (Fig. 1e). IF staining was performed in $90 \%$ confluent culture, which could explain the difference observed in flow cytometry analysis. ECs took up acetylated LDL $(95.3 \pm 3.7 \%, n=3)$ and were able to generate capillary-like tubular structures in the Matrigel angiogenesis assay (Fig. 1d, e).

For mesenchymal differentiation, flow cytometric analysis indicated that the majority of iNCC cells expressed HNK1 and P75 $(79.0 \pm 3.1 \%, n=3)$. Following mesenchymal terminal differentiation, essentially all the cells showed a typical MSC morphology, were positive for the majority of cell population which expressed CD73 and CD90 (95.1 $\pm 1.8 \%, n=3$ ) and CD105 (Fig. 1f). Also, iNCC staining revealed that the majority $(80.3 \pm 7.9 \%)$ of the cells were double positive for P75 and Vimentin. Similarly, most $(85.2 \pm 8.0 \%)$ iNCC-derived MSCs were double positive for CD73 and Vimentin (Fig. 1g). Additionally, $90.2 \pm 5.9 \%$ of these MSCs were positive for CD105 (Fig. 1g). Finally, we tested the ability of the iNCC-derived MSC to differentiate into osteogenic, chondrogenic, and adipogenic lineages. Figure $1 \mathrm{~h}$ shows representative, low-magnification bright-field images and photographs of cell culture wells, and negative controls, stained for Alizarin Red, Alcian Blue, and Oil Red, respectively, after 28-day exposure to the various MSC differentiation induction protocols.

\section{Liver organoid functional analysis}

Liver organoid (LO) formation was evaluated by assessing the tissue condensation rate (i.e., mesenchymal condensation rate) [7]. As seen in Fig. 2a, the rate of mesenchymal condensation was essentially identical for all cell lines and all experimental groups. No apparent morphological differences were observed in histological staining in the three cell lines and between groups (Fig. 2b shows representative H\&E images of the III group). No difference in cellular distribution was observed in IF tissue staining for hepatic, endothelial, and MSC markers (Additional file 1: Figure S1I). LO express important MRP1, an important basal membrane 

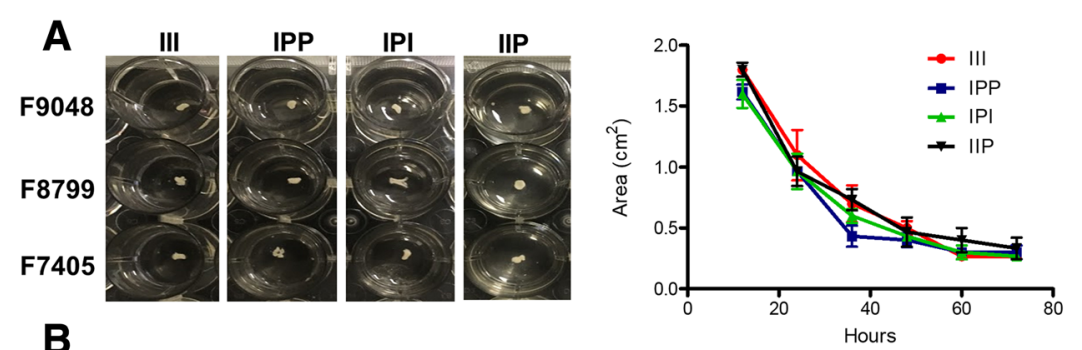

B

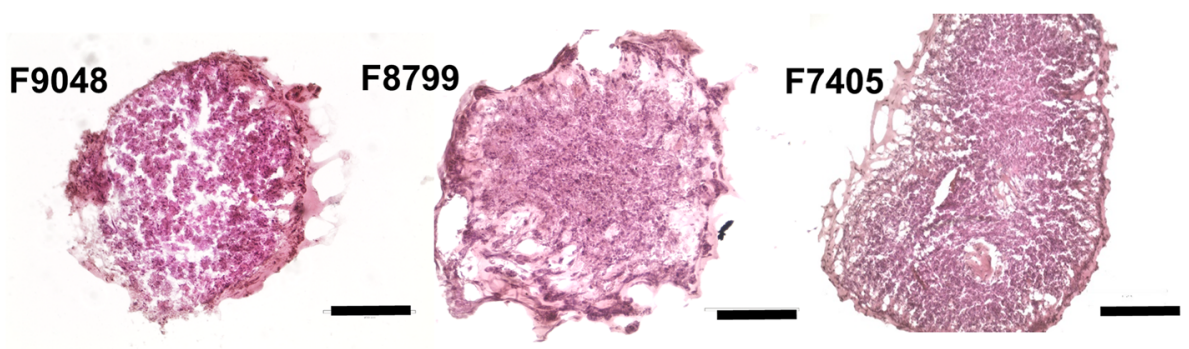

C
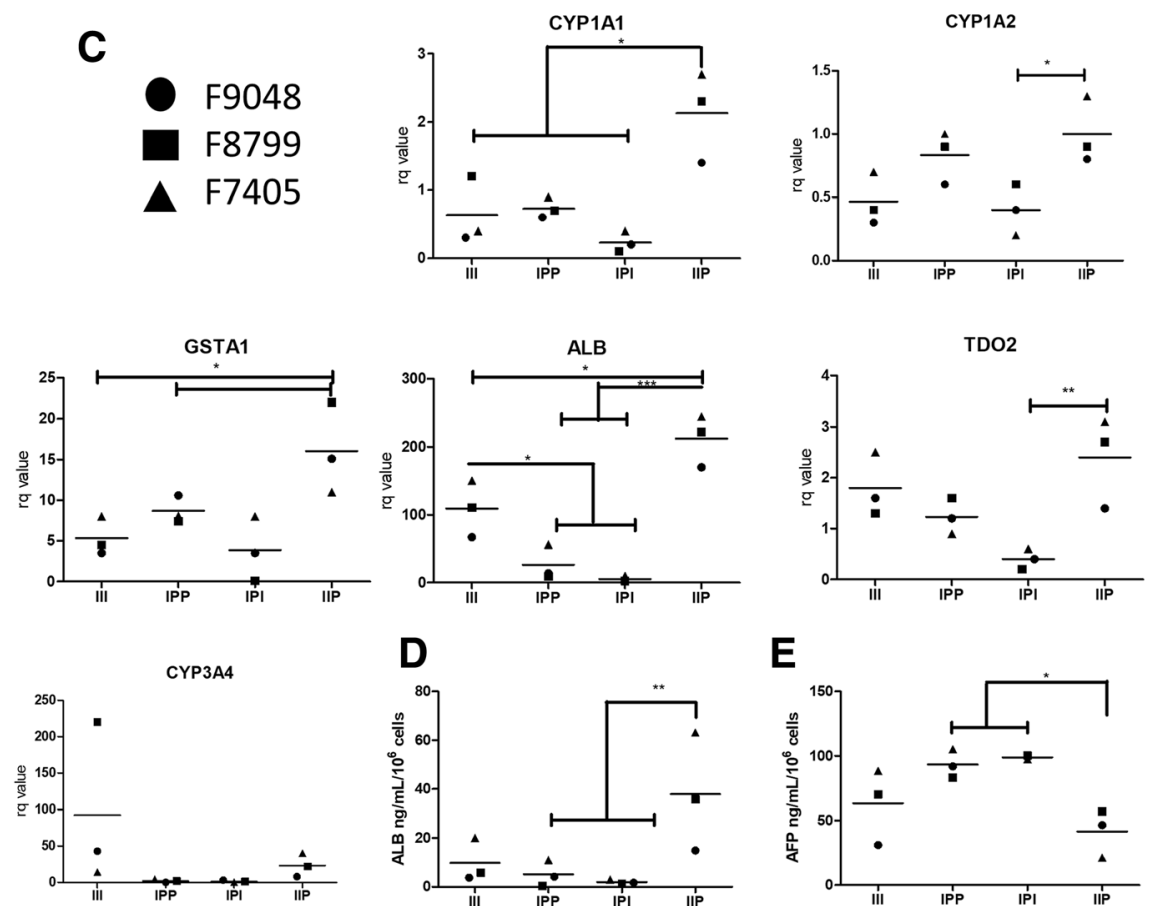

D

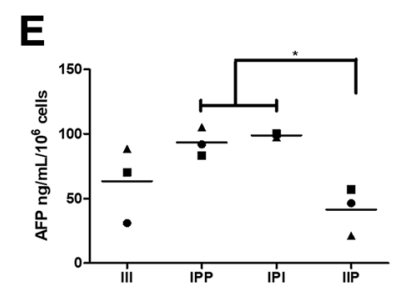

Fig. 2 Liver organoid functional analysis. a Representative image of liver organoid culture of all cell lines and tested conditions after $72 \mathrm{~h}$ of mesenchymal condensation in a 24-well plate and area analysis overtime $(n=3$, biological replicates; data displayed as mean \pm SEM). $\mathbf{b} \mathrm{HE}$ staining for III groups of all cell lines (bar $=50 \mu \mathrm{m}$ ). c RT-qPCR gene expression analysis of hepatic markers at day 12, normalized with 2D cultured iPS-derived hepatocyte at day 18 of differentiation protocol. d Normalized albumin secretion at day 6 and day 12. d Urea secretion at days 6 and 12. e AFP secretion at day $12(n=3$, biological replicates; data displayed as great mean and individual scatter plots; one-way ANOVA with Tukey's post hoc, ${ }^{*} p<0.05,{ }^{* *} p<0.01,{ }^{* *} p<0.01$ )

transporter, and were able to perform basolateral transportation, as shown by CDFDA staining (Additional file 1: Figure S1I). Analysis of gene expression by RT-qPCR after 12 days of $\mathrm{LO}$ culture revealed increased expression of important hepatic phase I xenobiotic biotransformation enzymes in IIP, such as CYP1A1 and CYP1A2, but not CYP3A4, and also an increased expression of the phase II enzyme GSTA1. Although CYP3A4 gene expression was not altered, enzymatic activity was significantly higher in group IIP (Additional file 1: Figure $\mathrm{S1G}$ ). The data also indicate an increased expression of important hepatic maturation markers, such as $A L B$ and TDO2. ELISA analysis of the LO culture supernatants revealed a significantly increased (6.7-fold) amount of 
secreted albumin by day 12 in the IIP group, as compared to other groups (Fig. 2d). Also, for the same time point, we observed a significant reduction of AFP secretion in the supernatants of group IIP, as compared to groups IPP and IPI (Fig. 2e). No statistical differences were observed at day 6 (Additional file 1: Figure S1E). We did not observe any differences in A1AT and LDH media production in all groups and in different time points (Additional file 1: Figure S1E).

\section{LO developmental pathway analysis}

Western blotting analysis of important cell signaling pathways related to liver development is displayed in Fig. 3a (experiment performed twice, independently). Densitometry analysis revealed significantly reduced activity (i.e., reduced ratio of phosphorylated/total protein) of SMAD2 (Fig. 3b) in III, as compared to IPP and IPI, but not to IIP. ERK1/2 activity was increased exclusively in III (Fig. 3c). $\beta$-Catenin expression was reduced in IIP, as compared to all groups (Fig. 3d). No statistically significant differences across all groups were found in the activities of Jagged-1 and SMAD1.5.7 (Fig. 3e, f). To confirm the western blotting findings, we performed an additional set of experiments using combinations of Wnt and TGF- $\beta$ agonists (i.e., CHIR99021 and TGF- $\beta 1$, respectively) and antagonist (i.e., DKK, WIF1, and SB431542, respectively) during LO maturation (Fig. 3g) for 10 days. Combinations of both agonists, $10 \mu \mathrm{M}$ CHIR99021 + $20 \mathrm{ng} / \mathrm{mL}$ of TGF-B1, significantly reduced the albumin gene expression, while the antagonists, 100 $\mathrm{ng} / \mathrm{mL}$ of DKK, $200 \mathrm{ng} / \mathrm{mL}$ WIF1 plus $10 \mu \mathrm{M}$ SB431542, significantly increased albumin gene expression (Fig. 3g).

\section{Proteomics}

Proteomic profiling revealed significant differences in LO groups at day 12 . Of the approximately 2100 proteins identified in each group, the vast majority of the protein IDs (2031) were identified (Fig. 4a). Principal

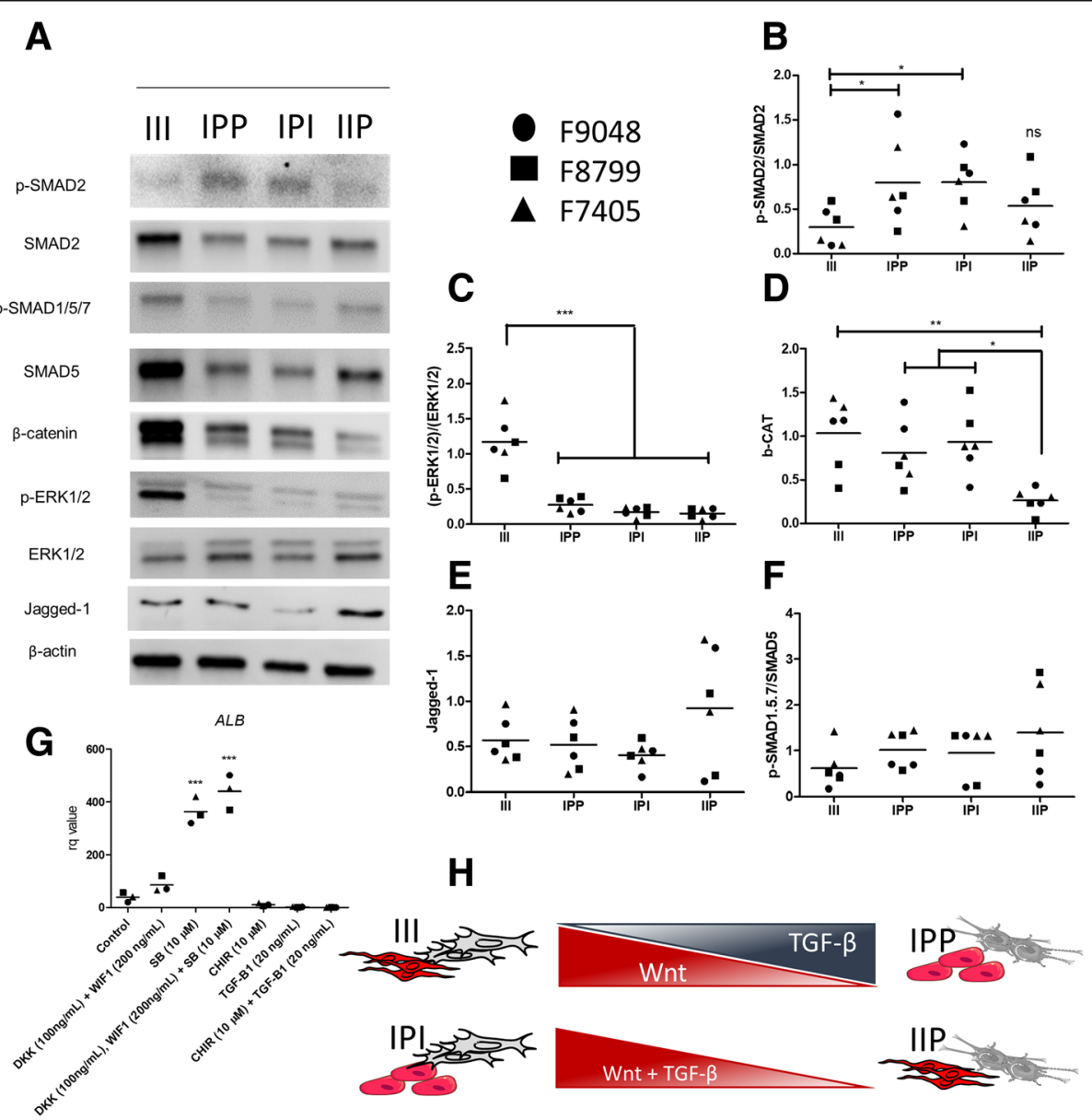

Fig. 3 Liver organoid cell signaling analysis. a Representative western blotting gels for all evaluated proteins (b) pSMAD2/SMAD2 analysis. c pERK1/2/ERK1/2 analysis. $\mathbf{d} \beta$-Catenin analysis. e Jagged-1 analysis. $\mathbf{f}$ p-SMAD1.5.7/SMAD1.5.7 analysis. g Albumin gene expression after IPP organoid culture in presence of TGF- $\beta$ and Wnt antagonist and agonist for 10 days. Data was normalized with untreated iPP organoid. $\mathbf{h}$ Graphical representation of western blotting results $(n=6$, biological replicates; data displayed as great mean and individual scatter plots; oneway ANOVA with Tukey's post hoc, ${ }^{*} p<0.05,{ }^{* *} p<0.01$, and $\left.{ }^{* * *} p<0.001\right)$ 


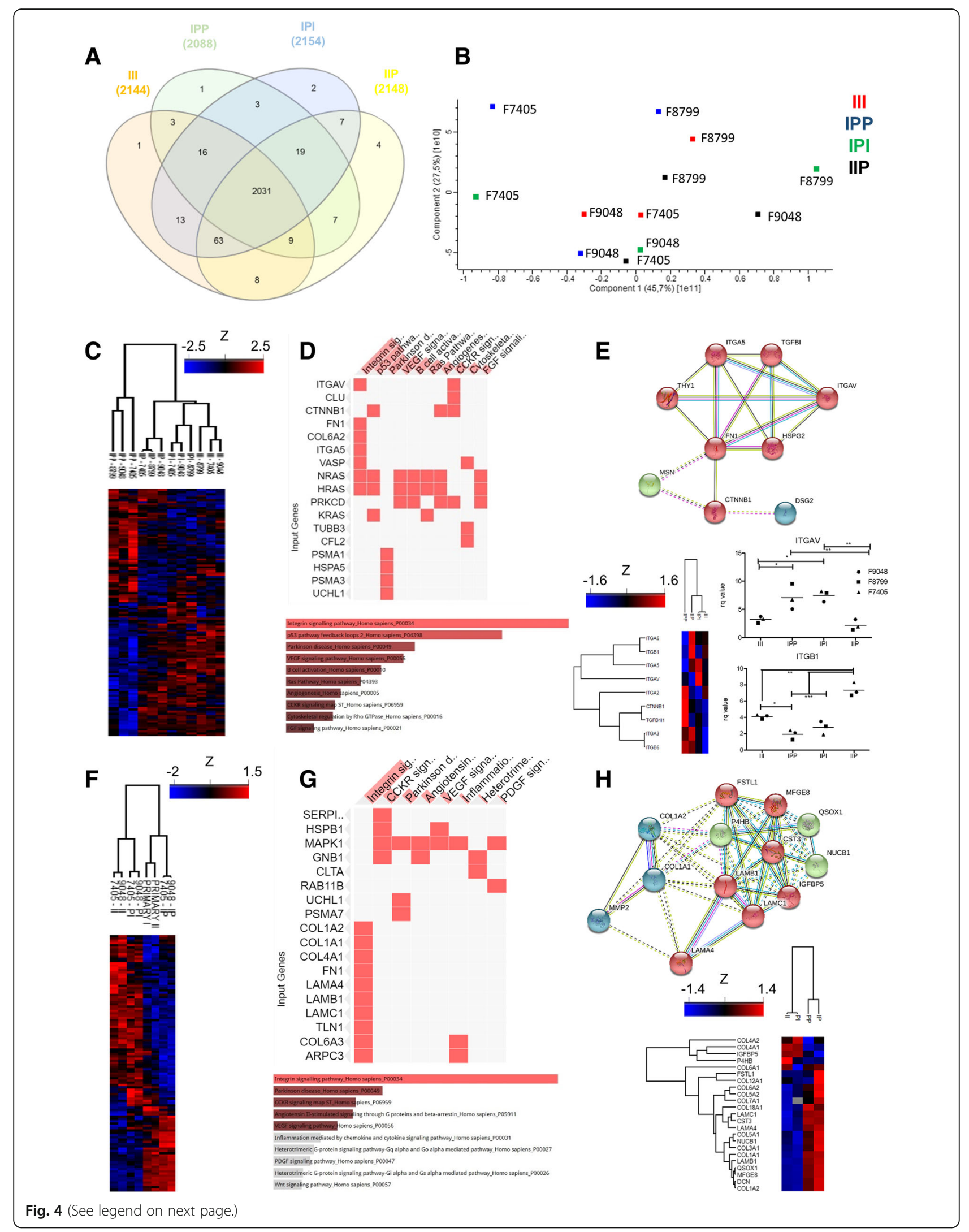


(See figure on previous page.)

Fig. 4 Liver organoid proteomic analysis. a Venn diagram of all identified proteins in all groups. b Principal component analysis of all tested samples. $\mathbf{c}$ Heat map of Z-scores from differentially expressed protein IDs in LO after one-way ANOVA test $(p<0.05)$. d Pathway enrichment analysis using EnrichR related to $\mathbf{c}$. e String interactome graph of protein from the list from $\mathbf{c}$, filtered by most enriched GO biological process, colored differently accordingly to k-means clustering. RT-qPCR for ITGAV and ITGBT ( $n=3$, biological replicates, normalized with 2D cultured iPSderived hepatocyte at day 18 of differentiation protocol; data displayed as great mean and individual scatter plots; one-way ANOVA with Turkey's post hoc, ${ }^{*} p<0.05$ and ${ }^{* *} p<0.01$ ). Filtered heat map for TGF, Wnt, and integrin signaling. $\mathbf{f}$ Heat map of Z-scores from differentially expressed protein IDs in LO secretome after one-way ANOVA test $(p<0.05)$. g Pathway enrichment analysis using EnrichR related to $\mathbf{f}$. $\mathbf{h}$ String interactome graph of the protein list from $\mathbf{f}$, filtered by most enriched GO biological process and filtered heat map for proteins related to ECM organization and IGF are colored differently accordingly to k-means clustering

component analysis showed the absence of clustering among all samples tested (Fig. 4b). Figure 4c shows a heat map of differentially expressed proteins, when applying ANOVA with $p<0.05$ threshold. Hierarchical clustering shows that the most different group was IPP, while the most similar among all groups were III and IPI. Figure $4 d$ highlights the integrin signaling as the most enriched pathway identified. Figure 4e shows the interactome from ANOVA-tested, differentially expressed proteins, filtered by the most enriched GO according to biological function. The two major clusters of nodes are located around FN1 and the integrin alpha subunits $\mathrm{V}$ and 5 , first shell of interactors, and TGFBI (i.e., TGF- $\beta$ induced protein) and CTNNB1 (i.e., $\beta$-catenin), corroborating western blotting findings. Gene expression analysis of ITGAV by RT-qPCR confirmed reduced expression in groups III and IIP, as opposed of what was observed in ITGB1. Secretome analysis of 2D co-culture of NPCs is displayed in Fig. 4f-h. Figure $4 \mathrm{f}$ shows the heat map generated by ANOVA with $p<0.05$ thresholds. Hierarchical clustering shows that IP is more similar to primary NPCs and that II is more similar to PI. Pathway enrichment analysis of the secretome of NPCs (Fig. 4g) highlights the role of integrins/extracellular matrix (ECM), TGF- $\beta$, and IGF, which are filtered and displayed in the heat map in Fig. 4h. The interactome of ANOVA-tested, differentially expressed proteins, filtered for ECM and IGF signaling, is shown in Fig. 4h.

\section{Discussion}

A major aim of the present study was to elucidate the role and efficacy of NPCs, derived either from iPS or from primary cell culture, in the development and functionality of LOs. Previous reports showed that it is possible to generate isogenic LOs using either primary or iPS-derived liver NPCs [8,9]. However, the impact of using such distinct NPC sources in LO maturation has never been addressed.

Here, we show that all human iPS cell lines used in the present study were characterized by flow cytometry and gene expression (Additional file 1: Figure S1A and B). We successfully differentiated all three iPS cell lines towards hepatoblasts, arterial ECs, and iNCC-derived
MSCs (Fig. 1 and Additional file 1: Figure S1C). The differential contribution, if any, of arterial, venous, or lymphatic ECs in the development of LO remains to be elucidated. Thus, we used iPS-derived arterial ECs, with commercially available HAECs as the correspondent adult cell line. For mesenchymal cells, we used iNCC-derived MSC, with dpMSC as the primary adult cell counterpart. Although there are many available protocols to differentiate iPS towards MSC, the vast majority of them lack proper comparison to the specific adult tissue MSC and are reported as a general embryonic or mesodermal MSC differentiation [17-19]. Since MSCs have tissuespecificity functionality, aiming to properly compare the use of primary versus iPS-derived MSC in LO maturation, we needed to compare matching tissue-derived primary MSC to iPS-differentiated tissue analog. Additionally, it is well known that iPS-derived MSCs obtained from different intermediated germ lines have different properties [18]. Our group recently reported a protocol to generate cranial facial MSC (obtained from iPS-derived neural crest cells) that closely resembles MSC located at the dental pulp [10]. Here, we were able to compare matching tissue specificity of MSCs (i.e., primary vs iPS-derived) in the LO maturation process.

We succeeded in deriving functional hepatoblasts, as assessed by the expression of HNF4A and AFP. The potential of our hepatoblasts to differentiate into hepatocyte was inferred from the expression pattern of mature hepatic markers such as UGT1A1 and CK18 and by PAS staining (Fig. 1b, c).

Arterial endothelial cell commitment was inferred from the expression of general endothelial markers, such as $C D 31$ and $V E C A D$, together with that of specific arterial EC markers such as NOTCH4 and the very low levels of expression of PDPN and EPHB4, which are markers of lymphatic and venous phenotype, respectively (Additional file 1: Figure S1B). The functionality of our iPS-derived ECs was demonstrated by their ability to take up acetylated LDL and to generate capillaries in Matrigel (Fig. 1d, e).

Neural crest differentiation was confirmed by the expression of HNK1 and CD75 (Fig. 1f). iNCC-derived MSC expressed stromal-mesenchymal markers such as CD105, CD90, and CD73 (Fig. 1f, g). Following in vitro 
induction, the mesenchymal differentiation was confirmed by the osteogenic staining with Alizarin Red (calcium deposits), chondrogenic staining with Alcian Blue (glycosaminoglycans), and adipogenic staining with Oil Red (lipid droplets) (Fig. 1h). This result indicates that we have successfully derived competent multipotent mesenchymal cells, and not fibroblasts.

LO generation was compared between all tested groups. No differences were observed in the mesenchymal condensation rate and in the morphology between all tested groups (Fig. 2a, b). Even though we noted some intrinsic variations between the tested cell lines, our RT-qPCR data at day 12 (Fig. 2c) revealed that important genes related to hepatic xenobiotic metabolism of phase I (i.e., CYP1A2 and CYP1A1) and II (i.e., GSTA1) were overexpressed in IIP. Even though CYP3A4 RT-qPCR data showed no significant differences, enzymatic activity levels were significantly higher in group IIP. These data suggest that the hepatic metabolic rate was higher in the presence of dpMSC associated with iPS-derived ECs (i.e., group IIP) and reduced in the presence of adult arterial ECs. ALB and TDO2 gene expression were also significantly elevated in group IIP, which suggests increased hepatic maturation. In addition, IIP produced more albumin at day 12 (Fig. 2c), with reduced AFP gene expression and secretion, as compared to IPI and IPP, but not to III. A concomitant increase in albumin and reduction of AFP secretion is one of the most important hallmarks of hepatocyte maturation [20-22]. The secretion of AA1T and LDH was not altered between the groups and at the time points tested (Additional file 1: Figure F).

To evaluate the influence of NPC in key signaling pathways relevant to LO formation, we performed a series of western blots (Fig. 4a) [23-29]. The protein activity analysis revealed that the III and IIP groups exhibited significantly lower activity of TGF- $\beta$ (Fig. $4 \mathrm{~b}$ ). Also, the IIP group showed significantly reduced Wnt activity (Fig. 4d), while the III group exhibited increased ERK1/2 activity (Fig. 4c). Activated ERK1/2 inhibits GSK3B through c-Met or IGF receptor signaling [29], which could explain high $\beta$-catenin in III. No differences were observed in the signaling of Notch and BMP4 (Fig. 4e, f). Figure $4 \mathrm{~h}$ compiles the information obtained from our western blot analyses. TGF- $\beta$ inhibition increased LO albumin production in vitro by inducing hepatoblast differentiation towards hepatocytes, thereby suppressing cholangiocyte differentiation [30]. Also, TGF- $\beta$ is positively correlated with lower $\mathrm{O}_{2(\mathrm{~g})}$ levels and activation of HIF1A during liver organogenesis [31]. In addition, Wnt signaling inhibition is known to control hepatocyte differentiation in 3D culture [32]. The combined inhibition of Wnt and TGF- $\beta$ significantly increases the expression of albumin (more pronounced by TGF- $\beta$ inhibition), as opposed of what was observed, when these two pathways were activated (Fig. 3g).

In order to confirm and evaluate the impact of previous western blotting analysis, we performed a proteomic profiling of the various LOs and of the NPC culture secretome. We observed intrinsic and differential protein expression patterns assigned by differential contribution of NPC to LO development (Fig. 4a-e). While sharing most of protein IDs (Additional file 1: Figure S1A), the tested NPC composition significantly influenced LO developmental pathways (Additional file 1: Figure S1B).

Most differentially enriched pathways were related to integrin signaling (Fig. 4c-e). The fibronectin receptor ITGAV (integrin receptor $\alpha \mathrm{V}$ ) was one of the major hits identified in our pathway enrichment analysis, as well as the integrin alpha subunit 5 (ITGA5). ITGAV expression is induced by TGF- $\beta$ and acts promoting epithelial-mesenchymal transition [33] and fibrosis [34]. Integrin subunits $\alpha 5$ and $\beta 1$ are necessary for bile duct epithelial tract formation during liver development [35]. Also, integrin $\beta 1$ is important for sustaining hepatocyte viability in native ECM and has been implicated in liver regeneration [36, 37]. Importantly, specific integrin subunit combinations during liver organogenesis, such as $\alpha 5 \beta 1$, help to generate the different hepatic structures and are influenced by surrounding sinusoids, vascular development, and local ECM [38]. In our secretome analyses of the NPC cultures, Decorin, a well-known endothelialproduced repressor of liver fibrosis and local inhibitor of TGF- $\beta$ [39] and c-Met [40], was significantly increased in group IIP (Fig. 4h). The reduced secretion of IGFBP5 by dpMSC (Fig. 4h), a MAPK signaling activator overexpressed during fibrosis [41], could explain the high ERK1/2 in III and reduced $\beta$-catenin in IIP. dpMSC produces more ECM, except for collagen type IV, but their role in LO maturation remains unclear.

Collectively, the expression of integrin $\beta 1$, but not $\alpha \mathrm{V}$, and reduced TGF- $\beta$ and Wnt signaling observed in the combination of iPS-derived EC and dpMSC, might explain the observed differences in hepatocyte function in various LOs. Our data suggests that high TGF- $\beta$ activity induced by HAEC (Fig. 3b) increased expression of ITGAV and induced ECM remodeling that impairs hepatocyte maturation. Additionally, we suggest that Wnt signaling repression in IIP is due to reduced secretion of IGFBP5 by dpMSC.

\section{Conclusion}

Our data indicates that reduced activity of TGF- $\beta$ and Wnt contributes for the increased albumin secretion and hepatic function observed in the combination of dpMSC and iPS-derived ECs as NPCs. These differential growth factor stimuli generate substantial changes in integrin and ECM profiles that regulate liver development. In 
translational terms, this work provides important insights for assessing future strategies to advance organoid technologies aiming at high-throughput drug screening platforms and regenerative therapy approaches.

\section{Additional file}

Additional file 1: Supplementary tables, methods, and figures (DOCX $1729 \mathrm{~kb}$ )

\begin{abstract}
Abbreviations
A1AT: Alpha 1 antitrypsin; AFP: Alpha fetoprotein; ALB: Albumin; BMP: Bone morphogenetic protein; CXCR4 : C-X-X motif receptor 4; CYP: Cytochrome P450; DKK: Dickkopf-related protein; dpMSC: Dental pulp-derived MSC; ECs: Endothelial cells; EGM: Endothelial growth media; ELISA: Enzyme-linked immunosorbent assay; FN1: Fibronectin; FOXA2: Forkhead box A2; GATA4: GATA binding protein 4; GSTA1: Glutathione S-transferase alpha 1; HAECs: Human aortic endothelial cells; HGF: Hepatocyte growth factor; HIF1A: Hypoxia induced-factor 1A; HNF4A: Hepatocyte nuclear factor 4 alpha; HNK1: Human natural killer-1; HUVEC: Human umbilical vein endothelial cells; IF : Immunofluorescence; IGF: Insulin-like growth factor; III: Organoid comprised by iPS-derived NPC; IIP: Organoid comprised by iPS-derived endothelial cells and dpMSC; IL-6: Interleukin 6; iNCC: iPS-derived neural crest cells; IPI: Organoid comprised by iPS-derived MSC and HAEC; IPP: Organoid comprised by adult primary NPC; iPS: Induced pluripotent stem cells; LDH: Lactate dehydrogenase; LDL: Low-density lipoprotein; MSC: Mesenchymal stem cells; NPC: Non-parenchymal cells; P75: Nerve growth factor receptor; PAS: Periodic acid of Schiff; PDPN: Podoplanin; RTqPCR: Reverse transcriptase quantitative polymerase chain reaction; SEM: Standard error of mean; TDO2: Tryptophan 2,3-dioxygenase; TGF$\beta$ : Transforming growth factor beta; TNF-a: Tumor necrosis factor alpha; UGT1A1: UDP glucuronosyltransferase family 1 member A1; VECAD: Vecadherin; WIF1: Wnt inhibitory factor 1
\end{abstract}

\section{Acknowledgements}

We would like to thank the funding agencies FAPESP/CEPID, CAPES, INCT, and CNPq. Ernesto Goulart and Luiz Carlos Caires-Junior are FAPESP grantees (grant numbers 2015/14821-1 and 2017/16283-2, respectively). We acknowledge the Mass Spectrometry Facility at Brazilian Biosciences National Laboratory (LNBio), CNPEM, Campinas, Brazil for their support on mass spectrometry analysis. We thank Valdemir Melechco Carvalho from Fleury Laboratory for the support on the proteomic experiments. Authors have no conflict of interest to disclaim. This work is dedicated to Raúl Marcel González Garcia, in memoriam.

\section{Authors' contributions}

EG designed the study under MZ mentorship and prepared the manuscript. EG, LCCJr, KATS, and BHAS performed the cellular experiments and analyzed the data. EG, LCCJr, KATS, GSK, CMM, AFA, and DO performed functional and proteomics analysis. EG, LCCJr, KATS, EC, JAG, SR, PIL, and MZ discussed the results and commented on the manuscript. All authors read and approved the final manuscript.

\section{Funding}

This work was supported by FAPESP (2013/08028-1).

\section{Availability of data and materials}

All data provided in this study are available within the article and its additional information files or accordingly to section "Data availability".

\section{Ethics approval and consent to participate}

The experimental procedures involving samples from human subjects are described in section "Ethics statement."

\section{Consent for publication}

Not applicable.

\section{Competing interests}

The authors declare that they have no competing interests.

\section{Author details}

${ }^{1}$ Human Genome and Stem-Cell Research Center (HUG-CEL), Department of Genetics and Evolutionary Biology, Institute of Biosciences, University of São Paulo, São Paulo, SP, Brazil. ²Brazilian Biosciences National Laboratory (LNBio), Brazilian Center for Research in Energy and Materials (CNPEM), Campinas, SP 13083-970, Brazil. 'Laboratory of Cellular Biology, Department of Pathology, Medical School, University of São Paulo, São Paulo, SP, Brazil. ${ }^{4}$ Department of Bioengineering, Temple University, Philadelphia, PA, USA. ${ }^{5}$ Department of Surgery, Medical School, University of São Paulo, São Paulo, SP, Brazil.

Received: 27 May 2019 Revised: 15 July 2019

Accepted: 31 July 2019 Published online: 15 August 2019

\section{References}

1. Takebe T, Sekine K, Enomura M, Koike H, Kimura M, Ogaeri T, Zhang R, Ueno $Y$, Zheng $Y$, Koike $N$, et al. Vascularized and functional human liver from an iPS-derived organ bud transplant. Nature. 2013;499:481-4.

2. Camp J, Sekine K, Gerber T, Loeffler-Wirth H, Binder H, Gac M, Kanton S, Kageyama J, Damm G, Seehofer D, et al. Multilineage communication regulates human liver bud development from pluripotency. Nature. 2017; 546:533-8.

3. Gordillo M, Evans T, Gouon-Evans V. Orchestrating liver development. Development. 2015;142:2094-108.

4. Shin D, Monga S. Cellular and molecular basis of liver development. Comprehensive Physiology. 2013;3(2):799-815.

5. Loh K, Ang L, Zhang J, Kumar V, Ang J, Auyeong J, Lee K, Choo S, Lim C, Nichane $M$, et al. Efficient endoderm induction from human pluripotent stem cells by logically directing signals controlling lineage bifurcations. Cell Stem Cell. 2014;14:237-52.

6. Asai A, Aihara E, Watson C, Mourya R, Mizuochi T, Shivakumar P, Phelan K, Mayhew C, Helmrath M, Takebe T, Wells J, Bezerra J. Paracrine signals regulate human liver organoid maturation from induced pluripotent stem cells. Development. 2017;144:1056-64.

7. Takebe T, Sekine K, Kimura M, Yoshizawa E, Ayano S, Koido M, Funayama S, Nakanishi N, Hisai T, Kobayashi T, et al. Massive and reproducible production of liver buds entirely from human pluripotent stem cells. Cell Rep. 2017;21: 2661-70.

8. Koui $Y$, Kido $T$, Ito T, Oyama H, Chen $S$, Katou $Y$, Shirahige $K$, Miyajima A. An in vitro human liver model by iPS-derived parenchymal and nonparenchymal cells. Stem Cell Reports. 2017;9:490-8.

9. Nie Y, Zheng Y, Ogawa M, Miyagi E, Taniguchi H. Human liver organoids generated with single donor-derived multiple cells rescue mice from acute liver failure. Stem Cell Res Ther. 2018;9:5.

10. Miller E, Kobayashi G, Musso C, Allen M, Ishiy F, de Caires L, Goulart E, GriesiOliveira K, Zechi-Ceide R, Richieri-Costa A, et al. EIF4A3 deficient human iPSs and mouse models demonstrate neural crest defects that underlie RichieriCosta-Pereira syndrome. Hum Mol Genet. 2017;26:2177-91.

11. Carnielli C, Macedo C, De Rossi T, Granato D, Rivera C, Domingues R, Pauletti B, Yokoo S, Heberle H, Busso-Lopes A, et al. Combining discovery and targeted proteomics reveals a prognostic signature in oral cancer. Nat Commun. 2018:9(1):359.

12. Kuleshov M, Jones M, Rouillard A, Fernandez N, Duan Q, Wang Z, Koplev S, Jenkins S, Jagodnik K, Lachmann A, et al. Enrichr: a comprehensive gene set enrichment analysis web server 2016 update. Nucleic Acids Res. 2016;8: W90-7.

13. Chen E, Tan C, Kou Y, Duan Q, Wang Z, Meirelles G, Clark N, Ma'ayan A. Enrichr: interactive and collaborative HTML5 gene list enrichment analysis tool. BMC Bioinformatics. 2013;15:128.

14. Fabregat A, Jupe S, Matthews L, Sidiropoulos K, Gillespie M, Garapati P, Haw $R$, Jassal B, Korninger F, May B, et al. The Reactome pathway knowledgebase. Nucleic Acids Res. 2018;4:D649-55.

15. Mi H, Thomas P. PANTHER pathway: an ontology-based pathway database coupled with data analysis tools. Methods Mol Biol. 2009;563:123-40.

16. Szklarczyk D, Morris J, Cook H, Kuhn M, Wyder S, Simonovic M, Santos A Doncheva N, Roth A, Bork P, et al. The STRING database in 2017: qualitycontrolled protein-protein association networks, made broadly accessible. Nucleic Acids Res. 2017;4:D362-8. 
17. Sheyn D, Ben-David S, Shapiro G, De Mel S, Bez M, Ornelas L, Sahabian A, Sareen D, Da X, Pelled G, Tawackoli W, Liu Z, Gazit D, Gazit Z. Human induced pluripotent stem cells differentiate into functional mesenchymal stem cells and repair bone defects. Stem Cells Transl Med. 2016;5(11):1447-60.

18. Eto S, Goto M, Soga M, Kaneko Y, Uehara Y, Mizuta H, Era T. Mesenchymal stem cells derived from human iPS cells via mesoderm and neuroepithelium have different features and therapeutic potentials. PLoS One. 2018;13(7):e0200790.

19. Kang R, Zhou Y, Tan S, Zhou G, Aagaard L, Xie L, Bünger CB, Luo L, Y. Mesenchymal stem cells derived from human induced pluripotent stem cells retain adequate osteogenicity and chondrogenicity but less adipogenicity. Stem Cell Res Ther. 2015;6(1):144.

20. Ang L, Tan A, Autio M, Goh S, Choo S, Lee K, Tan J, Pan B, Lee J, Lum J, et al. A roadmap for human liver differentiation from pluripotent stem cells. Cell Rep. 2018;22:2190-205.

21. Roll G, Willenbring H. Transplanted nonviable human hepatocytes produce appreciable serum albumin levels in mice. Stem Cell Res. 2010;5:267-70.

22. Peters D, Henderson C, Warren C, Friesen M, Xia F, Becker C, Musunuru K, Cowan C. Asialoglycoprotein receptor 1 is a specific cell-surface marker for isolating hepatocytes derived from human pluripotent stem cells. Development. 2016;143:1475-81.

23. Tanimizu N, Miyajima A. Notch signaling controls hepatoblast differentiation by altering the expression of liver-enriched transcription factors. J Cell Sci. 2004;117(Pt 15):3165-74.

24. Clotman F, Jacquemin P, Plumb-Rudewiez N, Pierreux CE, Van der Smissen P, Dietz HC, Courtoy PJ, Rousseau GG, Lemaigre FP. Control of liver cell fate decision by a gradient of TGF beta signaling modulated by Onecut transcription factors. Genes Dev. 2005;19(16):1849-54.

25. Frémin C, Ezan F, Guegan JP, Gailhouste L, Trotard M, Le Seyec J, Rageul J, Theret N, Langouët S, Baffet $\mathrm{G}$. The complexity of ERK1 and ERK2 MAPKs in multiple hepatocyte fate responses. I Cell Physiol. 2012;227(1):59-69.

26. Rossi JM, Dunn NR, Hogan BL, Zaret KS. Distinct mesodermal signals, including BMPs from the septum transversum mesenchyme, are required in combination for hepatogenesis from the endoderm. Genes Dev. 2001; 15(15):1998-2009.

27. Russell JO, Monga SP. Wnt/B-catenin signaling in liver development, homeostasis, and pathobiology. Annu Rev Pathol. 2018;13:351-78.

28. Manmadhan $\mathrm{S}$, Ehmer U. Hippo signaling in the liver - a long and everexpanding story. Front Cell Dev Biol. 2019;7:33.

29. Etnyre D, Stone AL, Fong JT, Jacobs RJ, Uppada SB, Botting GM, Rajanna S, Moravec DN, Shambannagari MR, Crees Z, Puri N. Targeting c-Met in melanoma. Cancer Biol Therapy. 2014;15(9):1129-41.

30. Ng S, Saeb-Parsy K, Blackford S, Segal J, Serra M, Horcas-Lopez M, No D, Mastoridis S, Jassem W, Frank C, et al. Human iPS derived progenitors bioengineered into liver organoids using an inverted colloidal crystal poly (ethylene glycol) scaffold. Biomaterials. 2018;182:299-311.

31. Ayabe H, Anada T, Kamoya T, Sato T, Kimura M, Yoshizawa E, Kikuchi S Ueno Y, Sekine K, Camp J, et al. Optimal hypoxia regulates human iPSderived liver bud differentiation through intercellular TGFB signaling. Stem Cell Reports. 2018;11:306-16.

32. Pettinato G, Ramanathan R, Fisher R, Mangino M, Zhang N, Wen X. Scalable differentiation of human iPSs in a multicellular spheroid-based $3 D$ culture into hepatocyte-like cells through direct Wnt/B-catenin pathway inhibition. Sci Rep. 2016;6:32888.

33. Mamuya FA, Duncan MK. aV integrins and TGF- $\beta$-induced EMT: a circle of regulation. J Cell Mol Med. 2012;16(3):445-55.

34. Henderson N, Arnold T, Katamura Y, Giacomini M, Rodriguez JD, McCarty JH, Pellicoro A, Raschperger E, Betsholtz C, Ruminski PG, et al. Targeting of av integrin identifies a core molecular pathway that regulates fibrosis in several organs. Nat Med. 2013;19(12):1617-24.

35. Tanimizu N, Kikkawa Y, Mitaka T, Miyajima A. a1- and a5-containing laminins regulate the development of bile ducts via $\beta 1$ integrin signals. J Biol Chem. 2012;287:28586-97.

36. Speicher T, Siegenthaler B, Bogorad RL, Ruppert R, Petzold T, Padrissa-Altes $\mathrm{S}$, Bachofner M, Anderson DG, Koteliansky V, Fässler R, Werner S. Knockdown and knockout of $\beta 1$-integrin in hepatocytes impairs liver regeneration through inhibition of growth factor signalling. Nat Commun. 2014;21(5):3862.

37. Pinkse GG, Voorhoeve MP, Noteborn M, Terpstra OT, Bruijn JA, De Heer E. Hepatocyte survival depends on beta1-integrin-mediated attachment of hepatocytes to hepatic extracellular matrix. Liv Int. 2004;24(3):218-26.
38. Shiojiri N, Sugiyama Y. Immunolocalization of extracellular matrix components and integrins during mouse liver development. Hepatology. 2004;40(2):346-55.

39. Baghy K, lozzo RV, Kovalszky I. Decorin-TGF $\beta$ axis in hepatic fibrosis and cirrhosis. J Histochem Cytochem. 2012;60(4):262-8.

40. Goldoni S, Humphries A, Nyström A, Sattar S, Owens RT, McQuillan DJ, lozzo RV. Decorin is a novel antagonistic ligand of the Met receptor. J Cell Biol. 2009;185(4):743-54.

41. Yasuoka H, Hsu E, Ruiz XD, Steinman RA, Choi AMK, Feghali-Bostwick CA. The fibrotic phenotype induced by IGFBP-5 is regulated by MAPK activation and Egr-1-dependent and -independent mechanisms. Am J Pathol. 2009; 175(2):605-15.

\section{Publisher's Note}

Springer Nature remains neutral with regard to jurisdictional claims in published maps and institutional affiliations.
Ready to submit your research? Choose BMC and benefit from:

- fast, convenient online submission

- thorough peer review by experienced researchers in your field

- rapid publication on acceptance

- support for research data, including large and complex data types

- gold Open Access which fosters wider collaboration and increased citations

- maximum visibility for your research: over $100 \mathrm{M}$ website views per year

At BMC, research is always in progress.

Learn more biomedcentral.com/submissions 